This is a self-archived version of an original article. This version may differ from the original in pagination and typographic details.

Author(s): Peltonen, Teemu; Ojajärvi, Risto; Heikkilä, Tero

Title: Mean-field theory for superconductivity in twisted bilayer graphene

Year: 2018

Version: Published version

Copyright: @ 2018 American Physical Society

Rights: In Copyright

Rights url: http://rightsstatements.org/page/InC/1.0/?language=en

Please cite the original version:

Peltonen, T., Ojajärvi, R., \& Heikkilä, T. (2018). Mean-field theory for superconductivity in twisted bilayer graphene. Physical Review B, 98(22), Article 220504(R).

https://doi.org/10.1103/PhysRevB.98.220504 


\title{
Mean-field theory for superconductivity in twisted bilayer graphene
}

\author{
Teemu J. Peltonen, Risto Ojajärvi, and Tero T. Heikkilä \\ Department of Physics and Nanoscience Center, University of Jyvaskyla, P.O. Box 35 (YFL), FI-40014 University of Jyvaskyla, Finland
}

(Received 11 May 2018; published 10 December 2018)

\begin{abstract}
Recent experiments show how a bilayer graphene twisted around a certain magic angle becomes superconducting as it is doped into a region with approximate flat bands. We investigate the mean-field $s$-wave superconducting state in such a system and show how the state evolves as the twist angle is tuned, and as a function of the doping level. We argue that part of the experimental findings could well be understood to result from an attractive electron-electron interaction mediated by electron-phonon coupling, but the flat-band nature of the excitation spectrum also makes the superconductivity quite unusual. For example, as the flat-band states are highly localized around certain spots in the structure, also the superconducting order parameter becomes strongly inhomogeneous.
\end{abstract}

DOI: 10.1103/PhysRevB.98.220504

\section{INTRODUCTION}

Experiments on strongly doped graphene [1-4] have shown that with proper preparations, graphene can be driven to the superconducting state. Such experiments indicate that the lack of superconductivity in undoped graphene is not necessarily due to a lack of an (effective) attractive electron-electron interaction with strength $\lambda$ that would drive graphene to be superconducting, but rather the small density of states (DOS) close to the Dirac point. Technically, in contrast to the Cooper instability for metals taking place with arbitrarily small $\lambda$, superconductivity in an electron system with a massless Dirac dispersion $\epsilon_{p}^{2}=v_{F}^{2} p^{2}$ and an energy cutoff $\epsilon_{c}$ has a quantum critical point [5] $\lambda_{c}=\pi \hbar^{2} v_{F}^{2} /\left(2 \epsilon_{c}\right)$ such that for $\lambda<\lambda_{c}$, mean-field superconductivity does not show up at any temperature. From this perspective, doping to a potential $\mu$ leads to an increased DOS, and thereby to a nonvanishing critical temperature $T_{c} \approx|\mu| \exp \left[-\left(\lambda_{c} / \lambda-1\right) \epsilon_{c} /|\mu|-1\right]$. An alternative approach would be to change the spectrum and increase the density of states close to the Dirac point. The extreme limit would be an approximately flat band of size $\Omega_{\mathrm{FB}}$, where the group velocity tends to zero. In such systems the critical temperature is a linear function of the coupling strength [6,7], $T_{c}=\lambda \Omega_{\mathrm{FB}} / \pi^{2}$, and a quite high $T_{c}$ can be expected even without extra doping [8-13].

Recent observations [14] of superconductivity in twisted bilayer graphene [TBG, see Fig. 1(a)] take place in systems where theoretical studies have predicted the occurrence of asymptotically flat bands [15-25]. There have been many suggestions of an unconventional superconducting state both for regular graphene [26,27] and for TBG [24,28-36], typically directly related with the Coulomb interaction, and in some cases related with nonlocal interactions. Here, we study the mean-field theory of superconductivity in such systems, starting instead from the hypothesis that the observations could be explained with the conventional electron-phonon mechanism from the flat-band perspective [37]. This hypothesis is justified on the grounds that the relative strength and the screening of attractive and repulsive interactions are uncertain. Furthermore, phonon-mediated attraction is considered a viable mechanism for the observed superconductivity on doped graphene [1-4,38].

In particular, we use the model of Refs. [15,20] for the spectrum of the twisted bilayer, add an on-site (leading to $s$-wave superconductivity) attractive interaction of strength $\lambda$, and evaluate the mean-field order parameter profile. We find that the order parameter, and along with it the mean-field critical temperature, have a similar nonmonotonous behavior with respect to the twist angle as in the experiments. We also predict the behavior of the density of states in the superconducting state, resulting from the peculiarities of the flat-band eigenstates and from the position dependence of the superconducting order parameter [Fig. 1(b)]. Even if our pairing interaction is quite simple, the resulting energy-dependent density

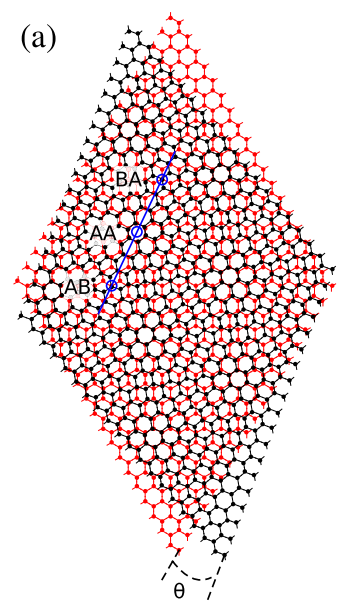

(b)

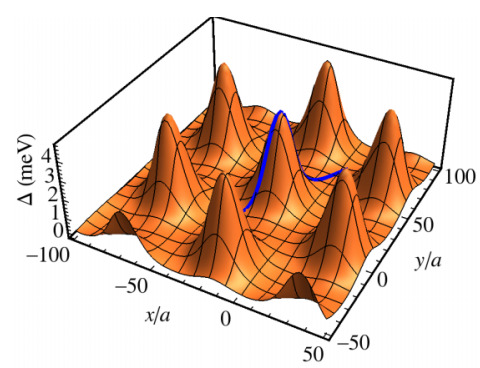

FIG. 1. (a) Twisted bilayer graphene and its moiré superlattice. The upper layer is rotated by an angle $\theta$ relative to the lower layer. (b) Position dependence of the self-consistent $\Delta$, shown here at $T=0$ for the magic angle $\theta=0.96^{\circ}$ and $\lambda=5 \mathrm{eV}^{2}$. In both figures also a line passing through high-symmetry points with $\mathrm{AB}, \mathrm{AA}$, and $\mathrm{BA}$ stacking is shown. 
of states is quite unusual. In addition, we show how doping away from the flat band eventually destroys superconductivity.

\section{NORMAL STATE}

We describe the normal state of TBG with the model of Refs. [15,20]. With this model, we can describe the twist angles $\theta$ at which the lattices $L$ and $L^{\theta}$ of the two graphene layers are commensurate, so that the system as a whole is periodic in the moiré superlattice $S L$. Here, we study only the simple commensurate structures, characterized by a single rotation parameter $m \in \mathbb{N}$, for which the rotation angle is given by

$$
\cos (\theta)=\frac{3 m^{2}+3 m+1 / 2}{3 m^{2}+3 m+1}
$$

According to Ref. [20], these structures approximate arbitrary commensurate structures. The primitive vectors of the superlattice $S L$ are given by $\boldsymbol{t}_{1}=m \boldsymbol{a}_{1}+(m+1) \boldsymbol{a}_{2}, \boldsymbol{t}_{2}=-(m+$ 1) $\boldsymbol{a}_{1}+(2 m+1) \boldsymbol{a}_{2}$, and the primitive vectors of the reciprocal superlattice $S L^{*}$ are $\boldsymbol{G}_{1}=\frac{4 \pi}{3\left\|\boldsymbol{t}_{1}\right\|^{2}}\left[(3 m+1) \boldsymbol{a}_{1}+\boldsymbol{a}_{2}\right]$, $\boldsymbol{G}_{2}=\frac{4 \pi}{3\left\|\boldsymbol{t}_{1}\right\|^{2}}\left[-(3 m+2) \boldsymbol{a}_{1}+(3 m+1) \boldsymbol{a}_{2}\right]$, where the lattice constant of the superlattice is $\left\|\boldsymbol{t}_{1}\right\|=\sqrt{3 m^{2}+3 m+1} a$ and the graphene lattice primitive vectors are $\boldsymbol{a}_{1}=(1, \sqrt{3}) a / 2$ and $\boldsymbol{a}_{2}=(-1, \sqrt{3}) a / 2$ with $a$ the lattice constant [15]. In the following, we assume that $\boldsymbol{G} \in S L^{*}$ belongs to the reciprocal superlattice, $\boldsymbol{k} \in \mathbb{R}^{2} / S L^{*}$ to the first Brillouin zone of the superlattice, and also that the corresponding sums and integrals are restricted to these sets.

In the normal state, TBG is described by a low-energy effective Hamiltonian [15]

$$
\mathcal{H}_{\rho \boldsymbol{k}}\left(\boldsymbol{G}, \boldsymbol{G}^{\prime}\right)=\left(\begin{array}{cc}
{\left[\hbar v_{F} \boldsymbol{\sigma}^{\rho} \cdot(\boldsymbol{k}+\boldsymbol{G}+\rho \Delta \boldsymbol{K} / 2)-\mu\right] \delta_{\boldsymbol{G}, \boldsymbol{G}^{\prime}}} & t_{\perp}^{\rho}\left(\boldsymbol{G}-\boldsymbol{G}^{\prime}\right) \\
t_{\perp}^{\rho}\left(\boldsymbol{G}^{\prime}-\boldsymbol{G}\right)^{\dagger} & {\left[\hbar v_{F} \boldsymbol{\sigma}_{\theta}^{\rho} \cdot(\boldsymbol{k}+\boldsymbol{G}-\rho \Delta \boldsymbol{K} / 2)-\mu\right] \delta_{\boldsymbol{G}, \boldsymbol{G}^{\prime}}}
\end{array}\right),
$$

where the matrix structure corresponds to the layer structure and $\rho \in\{+,-\}$ is the valley index with + corresponding to $\boldsymbol{K}$ and - to $\boldsymbol{K}^{\prime}=-\boldsymbol{K}$. Furthermore, each entry is a $2 \times 2$ matrix due to the sublattice structure in graphene. The diagonal terms in Eq. (2) describe the Dirac dispersion in the two layers and are diagonal also in $\boldsymbol{G}$. Here, $\boldsymbol{\sigma}^{\rho}=\left(\rho \sigma_{x}, \sigma_{y}\right)$. For the second layer we include the rotation $\theta$ so that $\boldsymbol{\sigma}_{\theta}^{\rho}=$ $e^{+i \theta \sigma_{z} / 2} \boldsymbol{\sigma}^{\rho} e^{-i \theta \sigma_{z} / 2} . \Delta \boldsymbol{K}=\boldsymbol{K}^{\theta}-\boldsymbol{K}$ is the relative shift of the Dirac cones between the layers. The coordinates correspond to those of layer 1 as measured from the $\boldsymbol{K}$ point, but shifted with a vector $+\Delta \boldsymbol{K} / 2$ for layer 1 and $-\Delta \boldsymbol{K} / 2$ for layer 2 . With this choice, the relative momentum $\boldsymbol{k}$ on both layers corresponds to the same absolute momentum. Furthermore, $\mu$ is the chemical potential describing the effect of doping, here taken to be equal in both layers.

The off-diagonal terms in the Hamiltonian describe the coupling between the two layers. The matrix element at valley $\rho$ between a state in sublattice $\alpha$ in layer 1 and a state in sublattice $\beta$ in layer 2 is

$$
t_{\perp}^{\rho, \alpha \beta}(\boldsymbol{G})=\frac{1}{N} \sum_{\boldsymbol{r}} e^{-i \boldsymbol{G} \cdot\left(\boldsymbol{r}+\delta_{\alpha B} \delta_{1}\right)} e^{i \rho \boldsymbol{K}^{\theta} \cdot \boldsymbol{\delta}^{\alpha \beta}(\boldsymbol{r})} t_{\perp}\left[\boldsymbol{\delta}^{\alpha \beta}(\boldsymbol{r})\right]
$$

where $\delta^{\alpha \beta}(\boldsymbol{r})$ is the horizontal displacement vector between the site at $\boldsymbol{r}$, sublattice $\alpha$ in layer 1 , and the nearest neighbor at sublattice $\beta$ in layer 2. $\boldsymbol{\delta}_{1}$ denotes one of the nearest-neighbor vectors connecting the graphene $\mathrm{A}$ and $\mathrm{B}$ sublattices. The sum is over the graphene A sublattice sites in the superlattice unit cell, and $N$ denotes the number of these sites. For the interlayer hopping energy $t_{\perp}(\boldsymbol{\delta})$ we use the same Slater-Koster parametrization as in Ref. [15]. Furthermore, we approximate the interlayer coupling by only including the matrix elements with $\boldsymbol{G} \in\left\{0,-\boldsymbol{G}_{1},-\boldsymbol{G}_{1}-\boldsymbol{G}_{2}\right\}$ (valley $\boldsymbol{K}$ ) or $\boldsymbol{G} \in\left\{0, \boldsymbol{G}_{1}, \boldsymbol{G}_{1}+\boldsymbol{G}_{2}\right\}$ (valley $\boldsymbol{K}^{\prime}$ ), since they are an order of magnitude larger than the rest.

For $\theta \approx 1^{\circ}$, the electronic dispersion becomes almost flat [19] and the group velocity $d \epsilon_{p} / d p$ tends towards zero. In Fig. 2 we plot the resulting normal-state dispersion
[Figs. 2(a)-2(c)] and the (local and total) density of states [Figs. 2(d)-2(i)] close to this "magic" angle. The exact value of this magic angle depends on the details of the hopping model. In our case it is around $0.96^{\circ}$, i.e., somewhat lower than what was found in Ref. [19]. However, the qualitative behavior of the local density of states (LDOS) is rather similar to the previous models. In particular, there are two closely spaced DOS peaks signifying the flattening of the bands. The local density of states is plotted along the line shown in Fig. 1, including three high-symmetry points with $\mathrm{AB}, \mathrm{AA}$, and BA stacking. These correspond to $r=-1 / 3,0$, and $1 / 3$, respectively.

\section{SUPERCONDUCTING STATE}

We assume that there is a local attractive interaction $\lambda_{\sigma_{1} \sigma_{2}}\left(\boldsymbol{r}_{1}, \boldsymbol{r}_{2}\right)=\delta_{\bar{\sigma}_{1} \sigma_{2}} \delta\left(\boldsymbol{r}_{1}-\boldsymbol{r}_{2}\right) \lambda$ with strength $\lambda$, which results [7] in an order parameter $\Delta_{\alpha i}(\boldsymbol{r})$ depending only on the center-of-mass coordinate $\boldsymbol{r}$ (and sublattice $\alpha$ and layer $i$ ). On the other hand, the classification of the order parameter symmetries to $s, d, f$, etc., is based only on the relative coordinate $\boldsymbol{r}_{1}-\boldsymbol{r}_{2}$, which in our model is always zero. Thus the symmetry is purely $s$ wave.

We do not consider the specific nature of the pairing interaction and for the purposes of this Rapid Communication it can be mediated by phonons or other bosonic modes This model disregards the retardation effects due to such a mechanism, but is a valid approximation to the more general Eliashberg approach for weak coupling $[39,40]$. That theory also shows that a direct Coulomb interaction, typically modeled via the Hubbard model, is less effective in reducing $\Delta$ than what could be naively expected, and should be included in the low-energy self-consistency equation as a Coulomb pseudopotential $[7,40,41] u^{*}=u /(1+u \alpha)$, where $u=U a^{2}$, $U$ is the Hubbard interaction constant, and $\alpha$ is a constant measuring the amount of renormalization due to the highenergy bands above the electron-phonon cutoff frequency 
$m=30\left(\theta=1.08^{\circ}\right)$

(a)

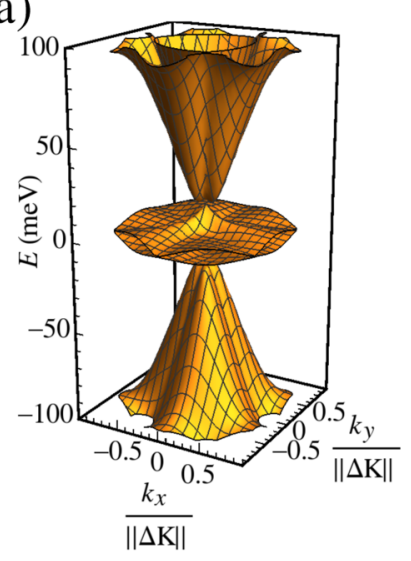

(d)

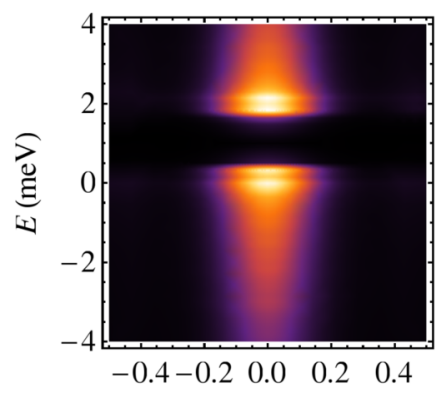

(g)

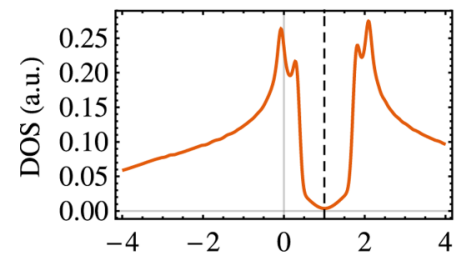

(j)

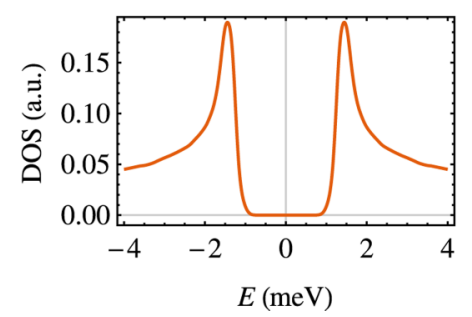

$$
m=34\left(\theta=0.96^{\circ}\right)
$$

(b)

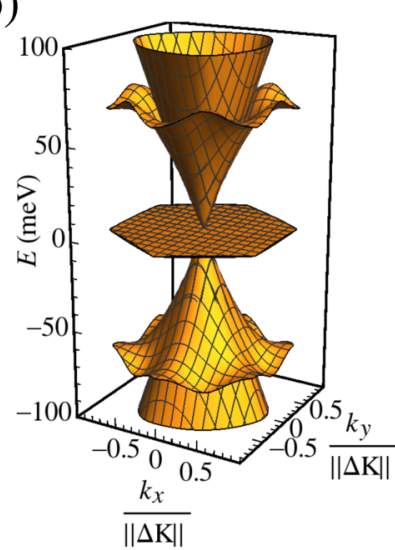

(e)

LDOS

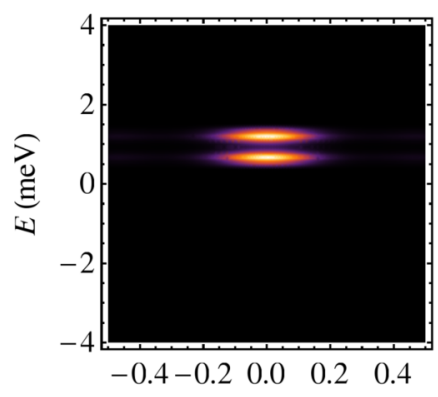

(h)
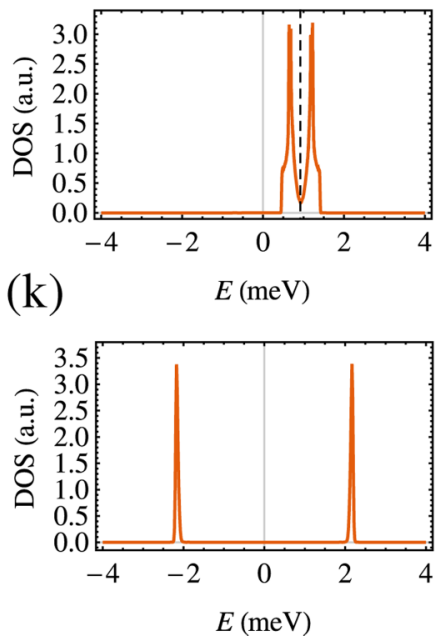

$m=38\left(\theta=0.86^{\circ}\right)$

(c)

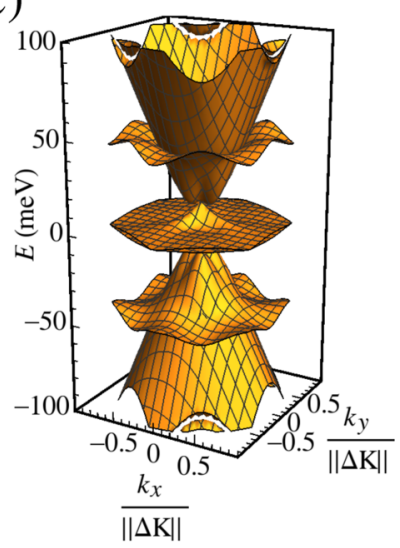

(f)

LDOS

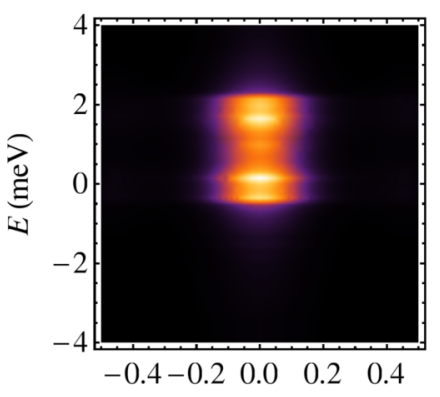

(i)
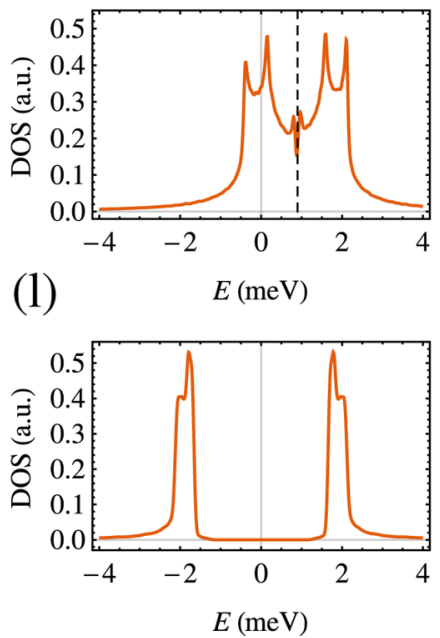

FIG. 2. (a)-(c) Normal-state dispersion, (d)-(f) local, and (g)-(i) total density of states for three different angles near the magic angle $\theta=0.96^{\circ}$ in the normal state. The bottom row (j)-(l) shows the corresponding total density of states in the superconducting state, in the case $T=0$ and $\lambda=5 \mathrm{eV} a^{2}$ and when doped to the point $\mu_{0}$ marked as a dashed line in (g)-(i).

$\omega_{D}$. For TBG we find from a simplified model [7] $\alpha \approx$ $0.2 \mathrm{eV}^{-1} a^{-2}$. Thus, a combination of electron-phonon and Coulomb interactions leads to an effective interaction strength $\lambda_{\text {eff }}=\lambda-u^{*}$. As long as $\lambda_{\text {eff }}>0$, there is a possibility for a superconducting state even if $u>\lambda$. For example, for $U=$ $5 \mathrm{eV}, u^{*}=2.5 \mathrm{eV} a^{2}$ is in the same regime as the value of $\lambda_{\text {eff }}$ in Figs. 3-5. Note that in what follows, we refer to this $\lambda_{\text {eff }}$ simply as $\lambda$.

Within a mean-field theory in the Cooper channel we find a self-consistency equation for a local superconducting order parameter [7]. Assuming that this order parameter shares the periodicity of the moiré superlattice, we find the selfconsistency equation

$$
\begin{aligned}
\Delta_{\alpha i}(\boldsymbol{G})= & \lambda \sum_{\rho, b} \sum_{\boldsymbol{G}^{\prime}} \int \frac{d \boldsymbol{k}}{(2 \pi)^{2}} \tanh \left(\frac{E_{\rho b \boldsymbol{k}}}{2 k_{B} T}\right) \\
& \times u_{\rho b \boldsymbol{k}, \alpha i}\left(\boldsymbol{G}^{\prime}\right) v_{\rho b \boldsymbol{k}, \alpha i}^{*}\left(\boldsymbol{G}^{\prime}-\boldsymbol{G}\right),
\end{aligned}
$$

where the band sum $b$ is calculated over the positive energy bands, $\alpha \in\{A, B\}$ is the sublattice index, $i \in\{1,2\}$ is the 
(a)

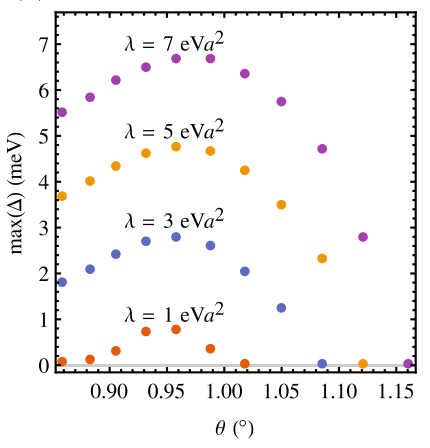

(b)

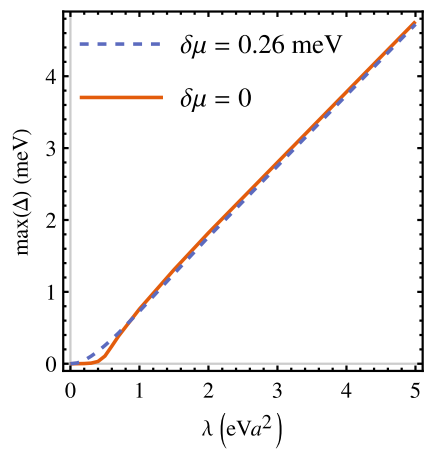

FIG. 3. Maximum of the position-dependent superconducting order parameter $\Delta(\boldsymbol{r})$ at $T=0$ as a function of (a) the rotation angle and (b) the coupling strength for $\theta=0.96^{\circ}$. In (b) we also show how doping to the DOS peak affects the small- $\lambda$ behavior.

layer index, and $u_{\rho b k}$ and $v_{\rho b k}$ are the eigenvectors of the Bogoliubov-de Gennes equation,

$$
\begin{aligned}
\sum_{\boldsymbol{G}^{\prime}}\left(\begin{array}{cc}
\mathcal{H}_{\rho \boldsymbol{k}}\left(\boldsymbol{G}, \boldsymbol{G}^{\prime}\right) & \Delta\left(\boldsymbol{G}-\boldsymbol{G}^{\prime}\right) \\
\Delta^{*}\left(\boldsymbol{G}^{\prime}-\boldsymbol{G}\right) & -\mathcal{H}_{\rho \boldsymbol{k}}\left(\boldsymbol{G}, \boldsymbol{G}^{\prime}\right)
\end{array}\right)\left(\begin{array}{l}
u_{\rho b \boldsymbol{k}}\left(\boldsymbol{G}^{\prime}\right) \\
v_{\rho b \boldsymbol{k}}\left(\boldsymbol{G}^{\prime}\right)
\end{array}\right) \\
\quad=E_{\rho b \boldsymbol{k}}\left(\begin{array}{c}
u_{\rho b \boldsymbol{k}}(\boldsymbol{G}) \\
v_{\rho b \boldsymbol{k}}(\boldsymbol{G})
\end{array}\right) .
\end{aligned}
$$

We solve this self-consistent order parameter with a few values of the interaction constant $\lambda$ and for a few different twist angles $\theta$ close to the magic angle. We include in the sum the energy levels closest to zero energy. We have checked that the results are not sensitive to the value of the energy cutoff, which we implement as a cutoff in the $b$ and $\boldsymbol{G}$ sums. For comparison between different angles, we measure the chemical potential from $\mu_{0}$, corresponding to the charge neutrality and marked in Figs. 2(g)-2(i) with a dashed line, by writing $\mu=\mu_{0}+\delta \mu$. The chemical potential shift $\mu_{0}$ is caused by the interlayer coupling. Unless otherwise stated, all the results concern the behavior at $\delta \mu=0$. The resulting total density of states is plotted in Figs. $2(\mathrm{j})-2(\mathrm{k})$, to allow for a comparison to the normal state. The corresponding local density of states (not shown) has the same localized structure as in the normal state, but the energy dependence is modified similarly as the total DOS. The effect of finite temperature on the superconducting DOS and LDOS happens solely via $\Delta(T)$, which is calculated below.

The maximum of the position-dependent $\Delta$, which according to numerics is equal in both layers and sublattices, is plotted in Fig. 3(a) for different values of the twist angle and for four different coupling strengths. The precise angle for the maximum depends a bit on the chosen coupling strength. Moreover, $\max (\Delta)$ is almost a linear function of $\lambda$ [see Fig. 3(b)], as appropriate for a flat-band superconductor [6]. This linearity is even more pronounced when the system is doped to the DOS peak at $\delta \mu \approx 0.26 \mathrm{meV}$. Far from the magic angle, the Fermi speed $v_{F}(\theta)$ increases so that the chosen $\lambda$ is below the critical value $\lambda_{c}$. This is why $\Delta$ vanishes for angles $\theta \gtrsim 1.1^{\circ}$.

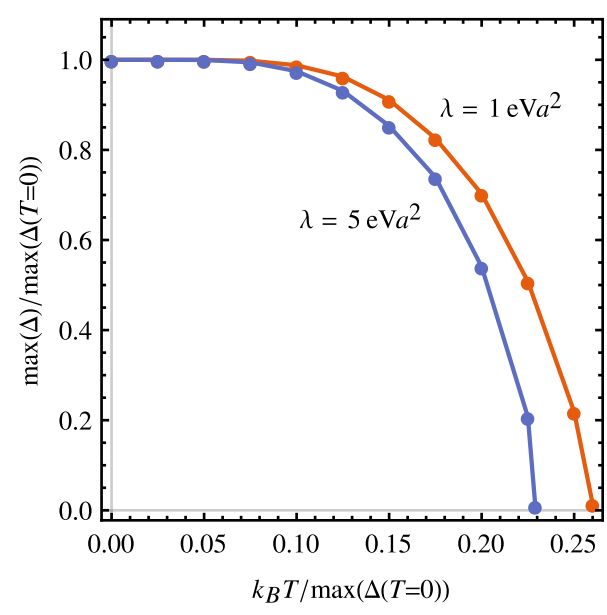

FIG. 4. $\max (\Delta)$ as a function of temperature in the case $\theta=$ $0.96^{\circ}$ for two values of $\lambda$, showing the approximate linear relation $k_{B} T_{c} \approx 0.25 \max [\Delta(T=0)]$ for the critical temperature. The dots are the calculated values and the lines are a guide to the eye.

We can analyze the resulting magnitude of $\Delta$ based on a flat-band result (assuming a position-independent $\Delta$ and $E_{\rho b k} \approx \Delta$ for an extreme flat band) according to which [7] $\Delta=\lambda \Omega_{\mathrm{FB}} / \pi^{2}$, where $\Omega_{\mathrm{FB}} \approx \Omega_{\text {moiré }}=8 \pi^{2} /\left(\sqrt{3}|| t_{1} \|^{2}\right)$. This yields $\Delta=1.3 \times 10^{-3} \lambda / a^{2}$ for $m=34$ corresponding to the magic angle. For comparison, a linear fit to the linear region in Fig. 3(b) gives $\max (\Delta)=-0.2 \mathrm{meV}+1.0 \times 10^{-3} \lambda / a^{2}$. The magnitude hence agrees very well with this simple model. Note that the precise values of these parameters especially for small $\lambda$ depend on the exact value of doping as shown below.

In Fig. 4 we show the temperature dependence of $\Delta$ for $m=34$, from which we may infer the approximate linear relation $k_{B} T_{c} \approx 0.25 \max [\Delta(T=0)]$ for the critical temperature. The prefactor is somewhat lower than for an extreme flat band with a constant $\Delta$, for which [7] $k_{B} T_{c}=\Delta / 2$. The difference is most likely explained by the nonvanishing bandwidth and the position-dependent $\Delta$ of our model. The maximum critical temperatures for the models calculated in Fig. 3(a) range from 3 to about $20 \mathrm{~K}$. The lower end of these values, calculated with $\lambda=1 \mathrm{eV} a^{2}$, is thus quite close to that found in Ref. [14].

We stress that the above result is the mean-field critical temperature; the observed resistance transition is most likely rather a Berezinskii-Kosterlitz-Thouless (BKT) transition $[42,43]$. Therefore, the mean-field $T_{c}$ gives an upper bound for the measured transition temperature. Furthermore, even the BKT transition temperature can be calculated from the mean-field superfluid weight [44]. The mean-field results are also relevant in that the DOS and LDOS can be experimentally measured by tunneling experiments and this depends on the structure and magnitude of mean-field $\Delta$ at temperatures below the BKT transition. Note that despite the flatness of the bands, the supercurrent can be nonvanishing in the case when the eigenstate Wannier functions overlap [45], as is the case for TBG.

Besides $\theta$ dependence, we can check how doping away from the center of the two DOS peaks affects the superconducting state. In Fig. 5(a) we plot the order parameter 

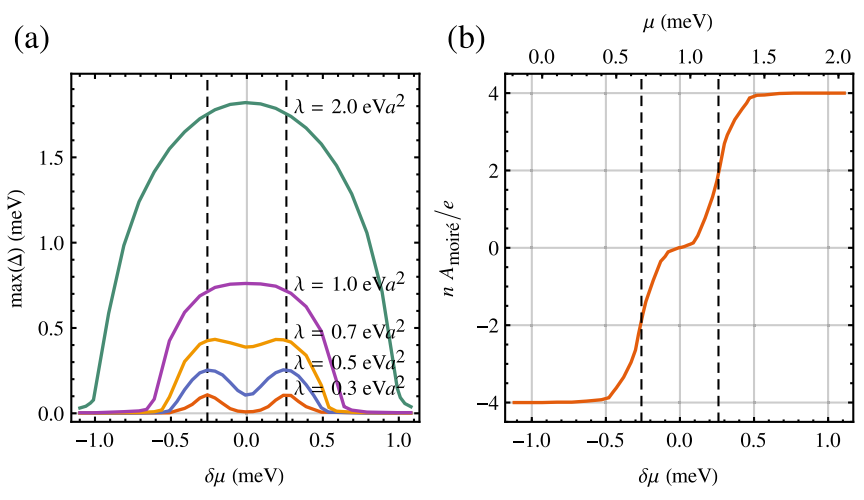

FIG. 5. Effects of electrostatic doping $\mu=\mu_{0}+\delta \mu$ for $\theta=$ $0.96^{\circ}$. (a) $\max (\Delta)$ vs chemical potential for various values of $\lambda$ at $T=0$. (b) Charge density in the normal state at $T=0$ as a function of chemical potential. The units of the charge density $n$ are $e / A_{\text {moiré }}$ where $e$ is the electron charge and $A_{\text {moire }}$ is the area of the moiré unit cell. In both figures the vertical dashed lines mark the location of the DOS peaks at $\delta \mu \approx \pm 0.26 \mathrm{meV}$.

$\max [\Delta(\delta \mu)]$ for different values of the doping $\delta \mu$ as measured from the charge neutrality point. Close to the magic angle, for $\lambda \gtrsim 1 \mathrm{eV} a^{2}$ the energy scale of superconductivity exceeds that of the normal-state dispersion, and hence the only effect of the doping is to move away from the flat-band regime, suppressing superconductivity [46]. For smaller values of $\lambda$, $\max (\Delta)$ is smaller than the bandwidth, and hence doping to the DOS peaks enhances superconductivity. Especially for $\lambda \lesssim 0.3 \mathrm{eV} a^{2}$ there are separate superconducting domes with doping levels close to the DOS peaks, which resembles the phase diagram in Ref. [14] for hole $(n<0)$ doping, apart

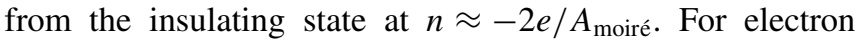
doping $(n>0)$, superconductivity is absent in the experiment, whereas our model is electron-hole symmetric. Since Ref. [14] uses charge density $n$ as a unit for the doping level while our theory is formulated in terms of the chemical potential $\mu$, for easier comparison we show the dependence between the charge density [7] and chemical potential in Fig. 5(b). From the figure we find that the DOS peaks correspond to approximately \pm 2 extra electrons per moiré unit cell.

\section{CONCLUSIONS}

Concluding, we find that a BCS-type mean-field model with a relatively weak attractive interaction constant possibly even due to electron-phonon coupling can explain the occurrence of superconductivity in twisted bilayer graphene. We also make a number of predictions concerning the mean-field superconducting state, in particular, the density of states and doping dependence. Our results form hence a checkpoint for further studies, that use a simplified picture of the TBG flatband states or consider mechanisms beyond the one in this Rapid Communication. Our results could also have relevance in explaining the observations of superconductivity in twisted interfaces of graphite [47-49].

Our mean-field theory fails to explain the insulator state [50] found experimentally in TBG at $n \approx \pm 2 e / A_{\text {moiré as well }}$ as the lack of superconductivity for electron doping [14,51]. However, the latter of these cannot be seen as a drawback of our model as in another experiment [52] some samples were found to be superconducting also on the electron-doped side, and thus it clearly depends on the samples and on the experimental setup. Regarding the insulator phase, it is plausible that the mean-field theory fails when the doping level corresponds to an integer number of electrons per superlattice unit cell. The biggest discrepancy is, however, most likely caused by the possible dependence of $\lambda_{\text {eff }}$ on the charge density, because the effect of the Coulomb interaction depends on charge screening. Within the flat-band model of Ref. [40], the case $\lambda_{\text {eff }}>0$ corresponds to a superconducting state, whereas for $\lambda_{\text {eff }}<0$ an insulating antiferromagnetic state is realized. Thus, by taking the chemical potential dependence of $\lambda_{\text {eff }}$ into account, it may be possible to describe both superconducting and insulating phases found in the experiment [14]. A detailed description would require generalizing Refs. [40,53] to the TBG case.

We point out that our simple BCS model disregards the strain effects in moiré bands, as well as the possible dependence of the interaction constant on the twist angle and doping level. Whereas such mechanisms may play a role in TBG, we believe that the simplest BCS-type mean-field superconductivity should also be considered as a viable effective model of the observations. Nevertheless, even in this case superconductivity would be highly exceptional, for example, because it can be so strongly controlled by electrostatic doping.

Note added. Recently, we became aware of Ref. [54], which addressed a similar BCS-type model as here, obtaining consistent results with this Rapid Communication. In addition to local interactions leading to $s$-wave superconductivity, they considered also nonlocal interactions opening the possibility to $d$-wave superconductivity. They found out that without including Coulomb repulsion the $s$-wave channel is more stable, having a higher $T_{c}$.

\section{ACKNOWLEDGMENTS}

This project was supported by Academy of Finland Key Project funding, and the Center of Excellence program (Projects No. 305256 and No. 284594). We acknowledge grants of computer capacity from the Finnish Grid and Cloud Infrastructure (persistent identifier urn:nbn:fi:research-infras2016072533).
[1] B. M. Ludbrook, G. Levy, P. Nigge, M. Zonno, M. Schneider, D. J. Dvorak, C. N. Veenstra, S. Zhdanovich, D.
Wong, P. Dosanjh et al., Proc. Natl. Acad. Sci. U.S.A. 112, 11795 (2015). 
[2] A. P. Tiwari, S. Shin, E. Hwang, S.-G. Jung, T. Park, and H. Lee, J. Phys.: Condens. Matter 29, 445701 (2017).

[3] J. Chapman, Y. Su, C. A. Howard, D. Kundys, A. N. Grigorenko, F. Guinea, A. K. Geim, I. V. Grigorieva, and R. R. Nair, Sci. Rep. 6, 23254 (2016).

[4] S. Ichinokura, K. Sugawara, A. Takayama, T. Takahashi, and S. Hasegawa, ACS Nano 10, 2761 (2016).

[5] N. B. Kopnin and E. B. Sonin, Phys. Rev. Lett. 100, 246808 (2008).

[6] T. T. Heikkilä, N. B. Kopnin, and G. E. Volovik, JETP Lett. 94, 233 (2011).

[7] See Supplemental Material at http://link.aps.org/supplemental/ 10.1103/PhysRevB.98.220504 for details of the derivation of the self-consistency equation, for the definition of the charge density, for a simplified model of the flat-band superconducting state, and for a calculation of the Coulomb pseudopotential, which includes Refs. [55,56].

[8] V. A. Khodel' and V. R. Shaginyan, Pis'ma Zh. Eksp. Teor. Fiz. 51, 488 (1990) [JETP Lett. 51, 553 (1990)].

[9] N. B. Kopnin, T. T. Heikkilä, and G. E. Volovik, Phys. Rev. B 83, 220503(R) (2011).

[10] E. Tang and L. Fu, Nat. Phys. 10, 964 (2014).

[11] V. J. Kauppila, F. Aikebaier, and T. T. Heikkilä, Phys. Rev. B 93, 214505 (2016).

[12] T. T. Heikkilä and G. E. Volovik, in Basic Physics of Functionalized Graphite, edited by P. D. Esquinazi (Springer, Berlin, 2016), pp. 123-143.

[13] T. Löthman and A. M. Black-Schaffer, Phys. Rev. B 96, 064505 (2017).

[14] Y. Cao, V. Fatemi, S. Fang, K. Watanabe, T. Taniguchi, E. Kaxiras, and P. Jarillo-Herrero, Nature (London) 556, 43 (2018).

[15] J. M. B. Lopes dos Santos, N. M. R. Peres, and A. H. Castro Neto, Phys. Rev. Lett. 99, 256802 (2007).

[16] E. Suárez Morell, J. D. Correa, P. Vargas, M. Pacheco, and Z. Barticevic, Phys. Rev. B 82, 121407(R) (2010).

[17] E. J. Mele, Phys. Rev. B 81, 161405(R) (2010).

[18] E. J. Mele, Phys. Rev. B 84, 235439 (2011).

[19] R. Bistritzer and A. H. MacDonald, Proc. Natl. Acad. Sci. U.S.A. 108, 12233 (2011).

[20] J. M. B. Lopes dos Santos, N. M. R. Peres, and A. H. Castro Neto, Phys. Rev. B 86, 155449 (2012).

[21] A. K. Geim and I. V. Grigorieva, Nature (London) 499, 419 (2013).

[22] D. Weckbecker, S. Shallcross, M. Fleischmann, N. Ray, S. Sharma, and O. Pankratov, Phys. Rev. B 93, 035452 (2016).

[23] S. Fang and E. Kaxiras, Phys. Rev. B 93, 235153 (2016).

[24] A. Ramires and J. L. Lado, Phys. Rev. Lett. 121, 146801 (2018).

[25] N. N. T. Nam and M. Koshino, Phys. Rev. B 96, 075311 (2017).

[26] B. Uchoa and A. H. Castro Neto, Phys. Rev. Lett. 98, 146801 (2007).
[27] R. Nandkishore, L. S. Levitov, and A. V. Chubukov, Nat. Phys. 8, 158 (2012).

[28] H. C. Po, L. Zou, A. Vishwanath, and T. Senthil, Phys. Rev. X 8, 031089 (2018).

[29] C. Xu and L. Balents, Phys. Rev. Lett. 121, 087001 (2018).

[30] C.-C. Liu, L.-D. Zhang, W.-Q. Chen, and F. Yang, Phys. Rev. Lett. 121, 217001 (2018).

[31] S. Ray and T. Das, arXiv:1804.09674.

[32] T. Huang, L. Zhang, and T. Ma, arXiv:1804.06096.

[33] J. F. Dodaro, S. A. Kivelson, Y. Schattner, X. Q. Sun, and C. Wang, Phys. Rev. B 98, 075154 (2018).

[34] G. Baskaran, arXiv:1804.00627.

[35] B. Roy and V. Juričić, arXiv:1803.11190.

[36] H. Guo, X. Zhu, S. Feng, and R. T. Scalettar, Phys. Rev. B 97, 235453 (2018).

[37] G. E. Volovik, JETP Lett. 107, 516 (2018).

[38] G. Profeta, M. Calandra, and F. Mauri, Nat. Phys. 8, 131 (2012).

[39] G. M. Éliashberg, Zh. Eksp. Teor. Fiz. 38, 966 (1960) [Sov. Phys. JETP 11, 696 (1960)].

[40] R. Ojajärvi, T. Hyart, M. A. Silaev, and T. T. Heikkilä, Phys. Rev. B 98, 054515 (2018).

[41] P. Morel and P. Anderson, Phys. Rev. 125, 1263 (1962).

[42] V. L. Berezinskii, Zh. Eksp. Teor. Fiz. 61, 1144 (1971) [Sov. Phys. JETP 34, 610 (1972)].

[43] J. M. Kosterlitz and D. J. Thouless, J. Phys. C: Solid State Phys. 6, 1181 (1973).

[44] A. Julku, L. Liang, and P. Törmä, New J. Phys. 20, 085004 (2018).

[45] S. Peotta and P. Törmä, Nat. Commun. 6, 8944 (2015).

[46] N. B. Kopnin and T. T. Heikkilä, in Carbon-based Superconductors: Towards High-T $T_{c}$ Superconductivity, edited by J. Haruyama (CRC Press, Boca Raton, FL, 2014), Chap. 9.

[47] P. Esquinazi, Pap. Phys. 5, 050007 (2013).

[48] A. Ballestar, J. Barzola-Quiquia, T. Scheike, and P. Esquinazi, New J. Phys. 15, 023024 (2013).

[49] M. Stiller, P. D. Esquinazi, J. Barzola-Quiquia, and C. E. Precker, J. Low Temp. Phys. 191, 105 (2018).

[50] B. Padhi, C. Setty, and P. W. Phillips, Nano Lett. 18, 6175 (2018).

[51] Y. Cao, V. Fatemi, A. Demir, S. Fang, S. L. Tomarken, J. Y. Luo, J. D. Sanchez-Yamagishi, K. Watanabe, T. Taniguchi, E. Kaxiras, and P. Jarillo-Herrero, Nature (London) 556, 80 (2018).

[52] M. Yankowitz, S. Chen, H. Polshyn, K. Watanabe, T. Taniguchi, D. Graf, A. F. Young, and C. R. Dean, arXiv:1808.07865.

[53] E. H. Hwang, R. Sensarma, and S. Das Sarma, Phys. Rev. B 82, 195406 (2010).

[54] F. Wu, A. H. MacDonald, and I. Martin, arXiv:1805.08735.

[55] A. H. Castro Neto, F. Guinea, N. M. R. Peres, K. S. Novoselov, and A. K. Geim, Rev. Mod. Phys. 81, 109 (2009).

[56] D. K. Efetov and P. Kim, Phys. Rev. Lett. 105, 256805 (2010). 